\title{
Health consequences of reproductive aging: a commentary
}

\author{
Siobán D. Harlow \\ Department of Epidemiology, University of Michigan, Ann Arbor, Michigan \\ Address for correspondence: Siobán D. Harlow, Department of Epidemiology, University of Michigan, 109 Observatory Street, \\ Ann Arbor, Michigan 48104. harlow@umich.edu
}

This commentary discusses the intersection of human ovarian and somatic aging. It argues for re-contextualizing estrogen's role in and impact on ovarian aging and, more broadly, on women's health, considering in particular the importance of timing, dose, and the broader endocrine milieu. Distinguishing between current clinical needs and optimizing women's future options, the paper outlines an approach to broadening the research agenda to better understand the role of ovarian aging in supporting the metabolic demands of longevity. Three overarching issues important to consider explicitly as we pursue research on the health correlates of reproductive aging are discussed, including implications of a lifespan approach, population diversity, and selection bias.

Keywords: ovarian aging; somatic aging; women's health

The papers in this section address the intersection of human ovarian and somatic aging and discuss emerging evidence that suggests a need to re-contextualize estrogen's role in and impact on ovarian aging and, more broadly, women's health. As articulated in these papers-timing matters, dose matters, and the broader endocrine milieu matters. It also matters whether our goal is to address current health needs with currently available options, for example, the problem that confronts physicians endeavoring to alleviate a woman's suffering with currently available options, or to optimize the options women may have in 10 or 20 years time.

To achieve the latter goal of optimizing future options, more explicit research efforts are needed:

(1) To develop an integrative understanding of the role of estrogen in physiology and health at different points across the life course;

(2) To place the physiologic impact of estrogen in the context of other critical and bioactive reproductive hormones (e.g., folliclestimulating hormone $[\mathrm{FSH}]$ );

(3) To weigh estrogen's importance relative to other aspects of physiology and in the context of environmental insults.

This commentary reflects on the contributions of three of the workshop presentations published here as well as on comments made in other presentations during this workshop session. I elaborate on three overarching issues important to consider more explicitly as we pursue research on the health correlates of reproductive aging-implications of a lifespan approach, population diversity, and selection bias.

An important question raised in the context of this session was whether an adaptive role of a low estrogen state might be articulated for the postmenopausal period as has been proposed for the period of lactation. ${ }^{1,2}$ For example, a biologic advantage has been proposed for hot flashes that benefits the newborn and for activation of the hypothalamic-pituitary-ovarian (HPO) axis to attenuate maternal stress responses and enhance metabolic and immune responses. ${ }^{1-3}$ Presenters questioned, however, whether the necessary compensatory mechanisms are established for a low estrogen state in the postmenopause to offset the negative consequences of loss of bone and increases in arterial tone, C-CRP, and LDL-C, etc. This excellent question requires more exploration.

Aging ova and the known exponentiation of risk of chromosomal disruptions in pregnancies after age $40^{4,5}$ may be the most parsimonious biologic explanation for ovarian senescence. Selection factors that enhance longevity may well not have 
ovarian origins. Alternatively, ovarian senescence may promote healthy aging by reducing the energy demands of repeated pregnancy and continued menstruation.

Workshop presenters argue that a low estrogen state is also adaptive in prolonging birth intervals and reducing fertility during periods of food scarcity. I noted with particular interest graphs reproduced from Emory Thompson et al. ${ }^{6}$ comparing fertility and survivorship in various populations and species. These graphs suggested that the !Kung, with the narrowest fertility curve and limited reproduction after age 35 , experience a survivorship of $50 \%$ at age $60-64$ and of $20 \%$ at age 70 , which is remarkable in a subsistence population. We might wish to consider further what this relationship suggests about environmentally moderated reproductive function and healthy aging.

Although not an expert on the evolutionary biology of aging literature, I would nonetheless ask whether we adequately understand the metabolic demands of longevity or the adaptive immune responses that promote longevity. From an evolutionary perspective we might inquire about the metabolic demands of taking on increased responsibility as a gatherer, ${ }^{7}$ how stamina or strength is best preserved, especially in the context of aging physiologic systems, and whether the relative increase in androgens, in fact, promotes survival.

The controversy in women's health regarding whether the postmenopausal low estrogen state should be construed as a deficiency state or an adaptive response to the demands of aging was highlighted by an analogy, and the critique of that analogy, made during the workshop between menopausal women and the witch in Hansel and Gretel. It was suggested that the physique of the old crone may be easily explained when framed through the lens of estrogen deprivation. Reconsidering this analogy, we are obliged to consider what alternative, perhaps environmental, explanations might be provided for this old woman's condition. Is her frailty fully determined by her postmenopausal state or might somatic aging contribute? The story, being set in the context of famine and dire poverty, suggests a potential role for malnutrition while vitamin $\mathrm{D}$ deficiency might also play a role given a life spent residing within the deep dark wood. The witch is, after all, a hardy survivor-the children's mother is dead (perhaps a maternal death) and their stepmother too does not survive the end of the story.

Alternatively, it may prove more productive to consider the differences between applying a construct of adaptation versus adjustment. If we can accept a value in limiting the reproductive period and the consequent necessity of a transition in the reproductive hormone milieu, how do we conceptualize a period of adjustment and what do we know about generating the optimal postreproductive physiologic state. The portrayal of aging as a state of menopausal estrogen deprivation can not in the end suffice, because the postmenopausal state is universal for all surviving women, yet decrepitude is not, and a place must be created for incorporating the fact of healthy aging. We have, perhaps, in much of our research failed to properly delineate bothersome symptoms of transition from chronic morbidity. Using the analogy of the stages of grief, we may need to consider more thoughtfully what constitutes normative adjustment versus an unresolved or morbid response.

Clinicians confront the suffering of a specific portion of the postmenopausal population. Hormone therapy (HT) helps ameliorate suffering. Yet the patient represents just one, relatively small, portion of the population distribution. Our scientific task is to consider more rigorously what combination of baseline risk, environmental modifiers, and patterns of reproductive aging are most indicative of risk.

One important lesson of the paradigm shift in the post-Women's Health Initiative (WHI) $\mathrm{era}^{8}$ is the importance of adopting a life course perspective and of balancing multiple competing risks within populations and individuals. Pinkerton and Stovall ${ }^{9}$ consider in careful detail the emerging clinical evidence for a differential impact of HT exposure given age at initiation and underlying disease status, particularly in the context of cardiovascular disease and dementia. The WHI and other data presented in this paper suggest the possibility of critical windows for intervention. Data emerging from the longitudinal cohort studies of midlife women, discussed by Ferrell and Sowers, ${ }^{10}$ also suggest that the midlife represents a critical period, setting the stage for women's long-term health across multiple dimensions. Traub and Santoro ${ }^{11}$ review the complex interrelationships that occur in the aging process between changes in the HPO axis and the 
somatotrophic axis. What remains unclear is the extent to which physiologic changes leading to cardiovascular disease, for example, originate in ovarian senescence or simply track with it. Further conceptualization is needed to articulate the specific meaning of a critical window as a period of adjustment, adaptation, or onset of decline. Is it that women must optimize opportunities to safely traverse this period of adjustment, or is it a window after which there is no safe haven? In either case, the unanswered questions include: how early might intervention be warranted and in whom?; what are the critical markers of preexisting abnormality?; and what might the risks of intervention be to women with a preexisting condition?

The epidemiologic literature generally supports an association between earlier age at menopause and mortality (all-cause and cardiovascular disease), ${ }^{12-16}$ with some evidence supporting an association also with cardiovascular disease incidence. ${ }^{17,18}$ However, these data are not fully consistent regarding the relative importance of premature menopause and surgical menopause in defining these risks. The extent to which risk in the latter subgroup is a consequence of early and/or abrupt ovarian senescence versus a consequence of the underlying health issue that led to the premature/early menopause/hysterectomy is underinvestigated and not resolved. Similarly, our understanding of the role of poor health in advancing age at menopause in general remains inadequate. Yet, evidence continues to build suggesting that poor health (diabetes, depression, heart disease) is associated with, or leads to, earlier age at menopause. ${ }^{19-23}$ Furthermore, several factors including body mass index, smoking, and socioeconomic status, are common correlates of menopause, menstrual characteristics across the lifespan, and mortality. ${ }^{19,20,22,24-26}$ Addressing these interrelationships will be critical to evaluating the timing of risk onset and the extent to which ovarian senescence does, or does not, play a causal role is defining women's risk status.

Ferrell and Sowers ${ }^{10}$ describe the longitudinal epidemiologic studies characterizing reproductive aging in population-based samples and discuss their contribution to our understanding of reproductive aging and ovarian senescence in populationbased samples of women. These studies typically capture all or part of the lifespan relevant to the "critical window" hypothesis and provide insights into factors associated with population variability in reproductive aging and, frequently, the dynamic interface between reproductive and somatic aging.

Recent data from these studies document the relatively late occurrence of the decline in estrogen in relation to other endocrine changes characteristic of the reproductive aging process including decline in anti-mullerian hormone (AMH), decline in inhibin and rise in FSH. This fact suggests the potential for more precisely delineating the timing of specific physiologic changes of somatic aging in relation to specific physiologic changes of reproductive aging as well as their relevant endocrine correlates. These authors specifically point to the interdependence of reproductive and somatic aging, stressing the importance of teasing out the impact of aging (i.e., aging of the hypothalamic-pituitary axis) on signals classically defined as those of reproductive aging (e.g., FSH).

Traub and Santoro ${ }^{11}$ summarize the emerging evidence of the role of estrogen in supporting the functional integrity of the somatotrophic axis during the menstrual cycle, its association with declines in insulin growth factor with aging and its relationship to cardiovascular risk and bone mineral density. They also summarize the evidence related to aging of the adrenal axis and its complex relationship to ovarian aging and chronic disease. They argue that low DHEAS levels have been associated with a range of health issues including cardiovascular disease, depression, and diabetes that previously have frequently been attributed to menopause and estrogen deficiency.

Of critical importance here is the need to establish:

(1) What role a woman's status at the onset of the menopausal transition plays in defining her risk profile;

(2) At what step in the reproductive aging process decrements in physiologic function are or are not initiated or accelerated; and

(3) Whether patterns or rates of change in reproductive function matter to health and wellbeing at the end of the transition.

Once these questions are answered, we may perhaps have an answer to the question "How important then is estrogen really?" 


\section{A lifespan perspective}

The critical window hypothesis has focused attention on the importance of midlife to health later in life. Yet, the midlife spans about one-fifth of a longlived individual's lifespan, and normal menopause itself may occur across a 20-year span from approximately age $40-60$. There is also substantial variability in the duration of the menopausal transition, especially the early transition. Longitudinal studies of reproductive age and midlife women have documented that poor risk profiles for a range of conditions, including low bone mineral density, often are established prior to the onset of the menopausal transition. ${ }^{26-28}$ We have yet to undertake studies with sufficient numbers of women enrolled in their peak reproductive or true late reproductive stage to provide a clear understanding of how baseline risk status segregates women's experience as they transition through the menopause or this presumed critical window. Thus, it is difficult to know whether the risk we observe at later life stages is dominated by baseline risk, for example, the failure to deposit adequate bone to weather the transition period. Most current studies enroll prevalent cohorts, that is women still menstruating, thus the at-risk women are frequently excluded. ${ }^{29,30}$

Although growing, the literature relating menstrual characteristics across the lifespan to other parameters of health and disease remains somewhat limited. Nonetheless, emerging data suggest that reproductive physiology tracks in particular ways across the life course and that the ovarian/somatic interlinkage also tracks in particular ways across the life course. For example,

(1) timing of menarche is associated with chronic disease risk but is not clearly correlated with peak reproductive menstrual characteristics ${ }^{31}$ or with age at menopause;

(2) long-normal menstrual cycles (30-31 days) are associated with higher fecundity ${ }^{32}$ and later age at menopause ${ }^{31}$;

(3) menstrual cycle length and variability are associated with chronic disease risk ${ }^{25}$; and

(4) timing of menopause is associated with chronic disease risk and mortality. ${ }^{13,14,16}$

Several questions about lifelong trajectories and the interlinkage between reproductive function and health across these trajectories remain, including:
(1) careful specification of the timing of cardiovascular insults and explication of what determines the ability of the cardiovascular system to weather the endocrine shift that is characteristic of menopause;

(2) explication of the health benefits of being postmenopausal, for example in terms of energy conservation, the importance of androgens to maintaining musculoskeletal health and whether there may be an evolutionary advantage to observed shifts in metabolism and to metabolic syndrome;

(3) what the relative health advantage of an osteoporotic versus osteoarthritic profile may be.

\section{Learning from population variability}

To date, the primary focus of the longitudinal population cohort studies of midlife women has been to describe the typical or dominant pattern of reproductive aging. However, these studies have the potential to provide important information on common variants from this typical or dominant pattern, as well as on genetic, behavioral, and/or environmental factors that might be associated with the population distribution of variant patterns. Increasing scientific focus on common variants of the reproductive aging process may facilitate understanding of the biologic tradeoffs that accompany these different trajectories and may provide insights into differential patterns of disease risk.

Gorrindo et al. ${ }^{33}$ have created an algorithm for typing women's lifetime menstrual histories into five categories: stable, stable but with greater variability, oscillating/erratic cycle lengths with a downward trend in cycle length over time, oscillating/erratic cycle lengths with a no downward trend in cycle length over time, and highly erratic. We are currently working on an approach for modeling cycle variability before and after the menopausal transition that allows for uncertainty in the typing of an individual woman's profile and permits us to relate profile types to covariates in the context of a regression model (Elliott, Huang, and Harlow, submitted for publication). Pinkerton and Stovall ${ }^{9}$ recommend increased focus on the subpopulation of women with surgical menopause. Oligomenorrheic women are another subgroup that warrants further research focus. As we learn more about variability in the reproductive aging process, it may be possible to define important subgroups in relationship to the 
probability of healthy aging and to clarify what the evolutionary advantage might be for each different population subgroup.

\section{Selection and bias}

In evaluating studies, whether they involve clinical populations or longitudinal population based samples, increased attention to selection factors and estimating bias is warranted. As noted earlier, most longitudinal cohort studies enroll prevalent cohorts with eligibility defined by presence of menses, excluding (or truncating) women who have already achieved their final menstrual period (FMP) at the time of enrollment. We have shown through simulations that bias can be substantial and standard errors severely underestimated in a naïve analysis which ignores this left truncation and/or left censoring and that this bias increases with increasing age of the prevalent cohort (Cain et al., submitted for publication). Research attempting to define critical thresholds for chronic disease risk may need to pay particular attention to issues of selection and bias if presence of underlying disease is associated with increased likelihood of being postmenopausal at a given age. Issues of generalizability, or lack thereof, may lead to mis-estimation of the association between reproductive and somatic aging. As noted by Ferrell and Sowers ${ }^{10}$ most of the cohort studies have captured relatively few hysterectomized women or women who are early or late transitioners.

Additional selection issues can arise when data are analyzed prior to all members of the cohort achieving the FMP or other markers of the menopausal transition. Current estimates of the timing of AMH decline, for example, reflect results from the early completers in the studies from which they are derived. Thus, published estimates of average time to FMP following AMH decline may well change as more women in these studies reach transition and menopause.

In summary, the papers in this section have raised important questions about the interrelationships between ovarian and somatic aging. Although knowledge of these interrelationships has increased in the last decade, a fundamental set of questions remains unanswered, that is to what extent, or in what context, is

(1) ovarian aging correlated with somatic aging, that is, it is an early marker of aging and should inform timely interventions;
(2) ovarian aging a trigger for somatic aging, consistent with the critical window hypothesis, such that our clinical goal should be to prolong ovarian function; or,

(3) ovarian aging triggered by somatic aging or underlying disease such that our clinical goal should be to treat the somatic disease.

\section{Conflicts of interest}

The author declares no conflicts of interest.

\section{References}

1. Lightman, S.L. et al. 2001. Peripartum plasticity within the hypothalamo-pituitary-adrenal axis. Prog. Brain Res. 133: 111-129.

2. Brunton, P.J., J.A. Russell \& A.J. Douglas. 2008. Adaptive responses of the maternal hypothalamic-pituitary-adrenal axis during pregnancy and lactation. J. Neuroendocrinol. 20: 764-776.

3. Mantzoros, C.S. 2000. Role of leptin in reproduction. Ann. N.Y. Acad. Sci. 900: 174-183.

4. Pellestor, F., T. Anahory \& S. Hamamah. 2005. Effect of maternal age on the frequency of cytogenetic abnormalities in human oocytes. Cytogenet. Genome Res. 111: 206212.

5. Hook, E.B. 1990. Chromosome abnormalities in older women by maternal age: evaluation of regression-derived rates in chorionic villus biopsy specimens. Am. J. Med. Genet. 35: 184-187.

6. Emory Thompson, M. et al. 2007. Aging and fertility patterns in wild chimpanzees provide insights into the evolution of menopause. Curr. Biol. 17: 2150-2156.

7. Kaplan, H. et al. 2010. Learning, menopause, and the human adaptive complex. Ann. N.Y. Acad. Sci. 1204: 30-42.

8. Writing Group for the Women's Health Initiative Investigators. 2002. Risks and benefits of estrogen plus progestin in healthy post-menopausal women. JAMA 288: 321333.

9. Pinkerton, J.V. \& D.W. Stovall. 2010. Reproductive aging, menopause, and health outcomes. Ann. N.Y. Acad. Sci. 1204: 169-178.

10. Ferrell, R.J. \& M.F. Sowers. 2010. Longitudinal epidemiologic studies of female reproductive aging. Ann. N.Y. Acad. Sci. 1204: 188-197.

11. Traub, M.L. \& N. Santoro. 2010. Reproductive aging and its consequences for general health. Ann. N.Y. Acad. Sci. 1204: 179-187.

12. Snowdon, D.A. et al. 1989. Is early natural menopause a biologic marker of health and aging? Am. J. Public Health 79: 709-714.

13. Jacobsen, B.K., I. Heuch \& G. Kvåle. 2003. Age at natural menopause and all-cause mortality: a 37-year follow-up of 19,731 Norwegian women. Am. J. Epidemiol. 157: 923929.

14. Cooper, G.S. et al. 2000. Age at menopause and childbearing patterns in relation to mortality. Am. J. Epidemiol. 151: 620623. 
15. Brett, K.M. \& G.S. Cooper. 2003. Associations with menopause and menopausal transition in a nationally representative US sample. Maturitas 45: 89-97.

16. Mondul, A.M., C. Rodriguez, E.J. Jacobs \& E.E. Calle. 2005. Age at natural menopause and cause-specific mortality. Am. J. Epidemiol. 162: 1089-1097.

17. Gordon, T., W.B. Kannel, M.C. Hjortland \& P.M. McNamara. 1978. Menopause and coronary heart disease. Ann. Intern. Med. 89: 157-161.

18. Colditz, G.A. et al. 1987. Menopause and the risk of coronary heart disease in women. N. Engl. J. Med. 316: 11051110.

19. Bromberger, J.T. et al. 1997. Prospective study of the determinants of age at menopause. Am. J. Epidemiol. 145: 124133.

20. Gold, E.B. et al. 2001. Factors associated with age at natural menopause in a multiethnic sample of midlife women. Am. J. Epidemiol. 153: 865-874.

21. Harlow, B.L., D.W. Cramer \& K.M. Annis. 1995. Association of medically treated depression and age at natural menopause. Am. J. Epidemiol. 141: 1170-1176.

22. Rödström, K. et al. 2003. Evidence for a secular trend in menopausal age: a population study of women in Gothenburg. Menopause 10: 538-543.

23. Dorman, J.S. et al. 2001. Menopause in type 1 diabetic women: is it premature? Diabetes 50: $1857-$ 1862.

24. Willett, W. et al. 1983. Cigarette smoking, relative weight, and menopause. Am. J. Epidemiol. 117: 651-658.

25. Harlow, S.D. \& S.A. Ephross. 1995. Epidemiology of men- struation and its relevance to women's health. Epidemiol. Rev. 17: 265-286.

26. Sowers, M.F, B. Shapiro, M.A. Gilbraith \& M. Jannausch. 1990. Health and hormonal characteristics of premenopausal women with lower bone mass. Calcif. Tissue Int. 47: 130-135.

27. MacMahon, B. et al. 1982. Age at menarche, probability of ovulation and breast cancer risk. Int. J. Cancer 29: 13-16.

28. Bainbridge, K.E. et al. 2002. Natural history of bone loss over 6 years among premenopausal and early post-menopausal women. Am. J. Epidemiol. 156: 410-417.

29. Dennerstein, L. et al. 2000. A prospective population-based study of menopausal symptoms. Obstet. Gynecol. 96: 351358.

30. Sowers, M.F. et al. 2000. Design, survey sampling and recruitment methods of SWAN: a multi-center, multiethnic, community-based cohort study of women and the menopausal transition. In Menopause: Biology and Pathobiology. Vol. 32. J. Wren, R.A. Lobo, J. Kelsey \& R. Marcus, Eds.: 175-188. Academic Press. San Diego.

31. Lisabeth, L., S.D. Harlow \& B. Qaqish. 2004. Marginal models for mean and variance: a new approach to modelling menstrual variation across the menopausal transition. $J$. Clin. Epidemiol. 57: 484-496.

32. Small, C.M. et al. 2006. Menstrual cycle characteristics: associations with fertility and spontaneous abortion. Epidemio$\log y$ 17: 52-60.

33. Gorrindo, T. et al. 2007. Lifelong menstrual histories are typically erratic and trending: a taxonomy. Menopause 14: 74-88. 